\title{
The duration of the cortisol awakening pulse exceeds sixty minutes in a meaningful pattern
}

\author{
Annika Benz ${ }^{\mathrm{a}}$, Maria Meier ${ }^{\mathrm{a}}$, Mara Mankin ${ }^{\mathrm{a}}$, Eva Unternaehrer ${ }^{\mathrm{a}}$, Jens C. Pruessner ${ }^{\mathrm{a}, \mathrm{b}, *}$ \\ ${ }^{\text {a }}$ University of Constance, Germany \\ ${ }^{\mathrm{b}}$ McGill University, Montreal, Quebec, Canada
}

Keywords:

Cortisol awakening response

CAR

Cortisol awakening pulse

CAP

Pulsatility

Measurement

Saliva

\begin{abstract}
A B S T R A C T
The cortisol awakening response (CAR) is a well-established biomarker for the integrity of the hypothalamicpituitary-adrenal (HPA) axis in healthy as well as clinical samples. Cortisol rise during the first 60 min after awakening is often used as a proxy of HPA axis regulation in health and disease. Ultradian pulsatility of cortisol is known to superimpose its circadian rhythmicity with the CAR being the first rise after awakening. However, the exact length of the complete first pulse (rise and fall) after awakening, as well as the association between the CAR, the complete first pulse, and successive ultradian pulses, has not yet been studied systematically.

Aim of this study was to investigate cortisol dynamics after awakening beyond the standard assessment duration of $60 \mathrm{~min}$ in order to assess the cortisol awakening pulse, in relation to ultradian rhythmicity of cortisol after awakening. In a sample of 51 healthy participants, salivary cortisol was collected for the first 270 min after awakening in intervals of $30 \mathrm{~min}$, on two separate days. Different parameters describing individual cortisol pulses over time with respect to inter- and intraindividual variations such as duration, amplitude and area under the curve (AUC) were calculated. Special emphasis was put on the first rise and fall after awakening, called the cortisol awakening pulse (CAP).

Mean duration of the CAP was 108 min with high interindividual differences. Duration of first and second pulse were associated with subject's sex and menstrual cycle status, with a longer first pulse and an attenuated second pulse in male and female subjects in the luteal phase, compared to female subjects in the follicular phase, or women taking oral contraceptives.

These results point to the potential usefulness of a longer sampling period for assessing ultradian pulsatility of cortisol in the morning, especially the CAP. To assess the complete pulse rather than the rise alone, measurement of cortisol levels after awakening for $120 \mathrm{~min}$ is recommended.
\end{abstract}

\section{Introduction}

The cortisol awakening response (CAR) enjoys a long history in psychoneuroendocrinological (PNE) research. One of the earliest mentions of a spike in cortisol after awakening in the morning stems from the work by Rivest et al. (1989), who investigated the relationship between the ultradian regulation of cortisol and melatonin in healthy men and noted that "... a burst of cortisol may follow awakening" (Rivest et al., 1989, p. 727). This suggested that cortisol levels rise and fall in response to waking, creating an individual spike with rapidly changing values over a short period of time. At the same time, clinical textbooks specified that the cortisol concentration in plasma taken early in the morning can range from 140 to $690 \mathrm{nmol} / \mathrm{l}$ without being considered conspicuous, or abnormal (Thomas, 1992). For PNE research, this was of little use as variations of 500\% prevent meaningful association with subgroups and psychological associations. Thus, in the mid 1990s, Clemens Kirschbaum and Dirk Hellhammer from Trier, Germany, had the idea to investigate patterns in cortisol concentrations in the morning more systematically, in the hope of replacing the 8 a.m. cortisol standard measure with something more consistent. The approach to measure cortisol in reference to awakening seemed logical as it would mark the earliest time point for measuring an individual's cortisol levels, regardless of absolute daytime. Since measurement of cortisol from saliva had just been introduced as a convenient and noninvasive method in PNE research (Kirschbaum and Hellhammer, 1989), it allowed for repeated testing in larger number of subjects. As it was unclear when cortisol levels would show highest consistency after awakening, the initial studies measured cortisol repeatedly with the

\footnotetext{
* Corresponding author at: University of Constance, Department of Psychology, Box 905, Universitaetsstrasse 10, 78457 Konstanz, Germany.

E-mail address: jens.pruessner@mcgill.ca (J.C. Pruessner).
} 
simple goal to identify the time point showing highest consistency. While those studies failed to demonstrate that one particular point in time was superior over another, two observations stood out: First, measuring cortisol in reference to awakening led to more consistency across days in the same individual as compared to the traditional 8 a.m. measure, and second, cortisol showed a significant increase in response to awakening, confirming Rivest's earlier finding through controlled experiments (Pruessner et al., 1997). As recently summarized by Stalder et al. (2016), publications on the CAR have been steadily on the rise since these first systematic studies and have consistently surpassed more than 100 articles per year since 2013. Currently, the CAR is an established marker of HPA axis integrity and shows stable associations with a number of clinical phenomena like depression (Dedovic and Ngiam, 2015), psychosis (Pruessner et al., 2013), and anxiety (Merswolken et al., 2012), to name just a few.

Although the rise of cortisol after awakening (i.e. the CAR) has been studied extensively over the last decades, several authors have highlighted the importance of the return of cortisol levels to baseline after their rise due to a stressor (e.g., Koolhaas et al., 2011; Scheurink et al., 1999). Koolhaas et al. could show that in rats, the corticosterone rise in response to a competitive social interaction is not inherently different in winners or losers; however, the recovery rate significantly differs between the two groups and hence provides additional information (Koolhaas et al., 2011). We suggest that this assumption is equally applicable for the cortisol dynamics in response to awakening. From researching the literature to find support for this model, it became clear, however, that the majority of studies assessing cortisol dynamics (A) restricted sampling protocols to the first $60 \mathrm{~min}$ after awakening to measure the rise in cortisol (i.e. the CAR), or (B) assessed a diurnal cortisol profile over the course of the day, such as a comprehensive large-scale analysis of diurnal cortisol profiles by Miller et al. (2007), without looking at the return of cortisol levels after their first rise in response to awakening. Thus, there seems to be a lack of studies looking at the 60 to $120 \mathrm{~min}$ time period past awakening. One single study standing out from the literature recently investigated the cortisol dynamics in $15 \mathrm{~min}$ intervals over the course of $8 \mathrm{~h}$ after awakening (Trifonova et al., 2013). As the sample size of this study was limited to 18 men, and the analyses focused on the comparison of ultradian cortisol pulsatility on saliva and plasma, we aim to build on these findings and measure the complete first cortisol pulse in response to awakening in a sample of healthy men and women. As the term CAR usually refers to the rise of cortisol in response to awakening, we want to distinguish the current approach from this well-established and valuable marker of HPA axis integrity. To this end, we introduce the term 'cortisol awakening pulse' (CAP) in the following to refer to the complete first pulse after awakening - comprising the rise, peak and fall of cortisol levels after awakening.

As such, the CAP shares characteristics with any pulsatile hormone release: it possesses an acrophase (distance from onset to peak, typically measured in time), a mesor (overall mean across time adjusted for rhythm), and an amplitude (distance from mesor to peak). If the oscillation is rhythmic, then the duration of the first pulse would also inform about the duration of the period (the distance in time from the start of one acrophase to the start of the following acrophase). These standard chronobiological terms have rarely been applied to cortisol dynamics in the morning, but it might be important to look at the CAP in the context of these descriptors (Fig. 1) as it has been shown that a dysregulation of the pulsatility of the HPA axis is associated with blunted cortisol responses to acute stress (Lightman and ConwayCampbell, 2010).

A recent article by Strüber et al. (2014) suggests that depending on the ratio of mineralocorticoid (MR) to glucocorticoid (GR) receptors in the hippocampus, the pulsatility of cortisol circadian rhythmicity might be affected, for example, in the aftermath of early traumatic events. Specifically, Strüber et al. argued that low maternal care together with the presence of the $\mathrm{L}$-allele of the serotonin receptor gene could lead to a change in the ratio of MR to GR in the hippocampus which in turn could lead to a flattened stress response and shortened ultradian pulses of cortisol. To establish the exact length of the CAP, one needs to determine not only the amplitude (i.e. the CAR), but also the total time interval that cortisol needs to return from the CAR peak back to basal level. It further implies that the CAP is associated with subsequent pulses, i.e., a flatter CAP would be followed by a flatter second pulse.

To sum up, measuring for $60 \mathrm{~min}$ is in line with the guidelines for assessing the CAR (Stalder et al., 2016), and has produced many interesting findings over the years. Yet, expanding the standard CAR monitoring protocols temporally and thus, assessing the CAP, might potentially offer new insights into HPA (dys)regulation and its link to the pathogenesis of stress-related disorders (Lightman and ConwayCampbell, 2010). As Miller et al. (2007) point out in conclusion of a comprehensive analysis of a meta-dataset of HPA responsivity in association with chronic stress, future research needs to focus on the decline of HPA activity supplementing well-established associations of cortisol increase after awakening.

Aim of the current investigation was therefore to investigate cortisol dynamics beyond the first $60 \mathrm{~min}$ after awakening (i.e. the CAR) and to describe and evaluate the CAP as a potential additional marker of HPA axis integrity. Thus, we hypothesized that the CAP (as defined by the time interval from the start to the first cortisol peak and the subsequent trough) would be significantly longer than $60 \mathrm{~min}$ as most CAR studies report cortisol levels not back at baseline after $60 \mathrm{~min}$.

Further, if the model of Strüber et al. (2014) is correct, then amplitude and duration of the CAP should be systematically associated with subsequent ultradian pulsatility. Thus, our second hypothesis was that the CAP would be a significant predictor of subsequent pulses, and that the higher the amplitude and the longer the duration of the CAP, the higher the amplitude and the longer the duration of the second pulse.

Finally, as previous studies have shown significant associations of sex, and associated hormonal status with the CAR, we wanted to determine whether sex and hormonal status would be associated with amplitude and duration of the CAP. As the majority of our sample consisted of young adult women, we estimated the menstrual cycle phase from self-report and assessed the use of oral contraceptives (OC) of female participants, in comparison to men, to determine if and how these factors are related to amplitude and duration of the CAP. Based on findings with the CAR, we hypothesized that we would find these associations with the CAP as well.

\section{Methods}

\subsection{Procedure}

Data for this study was collected in a sample of students from the University of Constance, Germany, from December 2017 until February 2018. After subjects completed a prescreening and psychological measurements (see 2.3), those eligible for the study were instructed to provide salivary samples on two weekdays for $270 \mathrm{~min}$ after awakening, at intervals of 30 min (mean interval between days of sample collection $=4.94$, range: $1-8$ days), resulting in ten saliva samples per day and two observations per participant. Subjects were instructed to collect these samples over the course of the first $4 \frac{1}{2}$ hours after awakening while following their normal daily routine. They were asked to record the time of sample collection, as well as the time and nature of any unusual activities (e.g. sleep disturbance, arguments with peers, physical activity) on a protocol sheet. Participants were instructed to rinse their mouth with water prior to each sample collection. The importance of adhering to the study protocol and following the exact sampling times and guidelines for saliva collection as outlined by Stalder et al. (2016) was emphasized. 

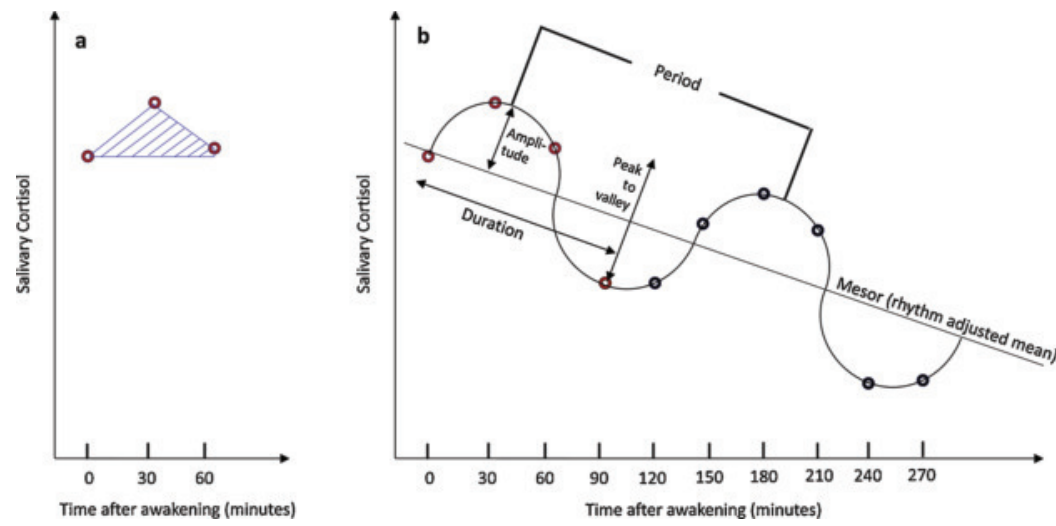

Fig. 1. Illustration of (a) traditional computation of the cortisol awakening response (CAR) as the AUCi(CAR) and (b) additional parameters computed individually per subject taking the complete cortisol awakening pulse (CAP; depicted by the first four red circles) into account. From the complete pulse, AUCi(CAP), amplitude, peak-to-valley value, and duration can be computed. (For interpretation of the references to colour in this figure legend, the reader is referred to the web version of this article).

\subsection{Participants}

A total of 55 subjects participated in this study. Participants were prescreened to exclude those with current, or any history of medical or psychiatric illness known to affect HPA axis activity (exclusion of $n=2$ ). In addition, symptoms of moderate to severe depression (as suggested by Beck Depression Inventory [BDI-II]: BDI > 19, Beck et al., 1996), being over- or underweight $(17.5<$ body mass index (BMI) < 29.9; Gwirtsman et al., 1989; Kumari et al., 2010), current pregnancy (Mastorakos and Ilias, 2003), medication known to affect HPA axis (exclusion of $n=1$ ), or drug use, served as additional exclusion criteria. Individual observations, defined as a single day of sample collection, were excluded in case of non-compliance to the study-protocol (exclusion of $n=1$ due to heavy exercise). Finally, data of 51 healthy subjects (10 men, mean age $=24.90 \pm 3.00$ years, range $=20-30$ years; 41 women, mean age $=21.32 \pm 3.28$ years, range $=18-32$ years) was analyzed.

Self-reports from female participants on use of OC and menstrual cycle phase were used to estimate hormonal status. Women reported the first day of their menses from the last two cycles and provided information about their regular cycle length so that estimates for their menstrual cycle phase at the two sampling days could be determined. Female participants without OC use provided information on the beginning of their last menstrual cycle (M1(latest)) and their average cycle duration $(m C D)$. An average luteal phase length of about 14 days was assumed as suggested by Lenton et al. (1984). The predicted day of ovulation (Dov) was estimated as

Dov $=$ mCD -14

The menstrual phase of a participant was estimated according to the following rules: If the number of days since the M1(latest) was greater than the Dov, participants were estimated to be in the luteal phase. Otherwise, they were estimated to be in the follicular phase. Women who reported to have no or only irregular menstrual cycle were excluded from analysis including this variable.

The study was approved by the Ethics Committee of the University of Constance and written informed consent was obtained from each participant. Subjects received financial compensation ( $€ 25$ ) or experiment participation credit $(2.5 \mathrm{~h})$ for study participation.

\subsection{Measurements}

\subsubsection{Psychological \& demographic assessment}

All demographic and study variables needed to determine eligibility (inclusion / exclusion criteria) and for further data analysis, including sex, age, height and weight, mental and medical health status, use of drugs or medication, menstrual cycle information, were collected as part of the prescreening via online self-report in-house questionnaires. Menstrual cycle phase information was additionally provided by female participants on both study days via paper-pencil self-report in the protocol sheet. Symptoms of depression and anxiety were assessed to control for their possible impact on HPA axis function. Severity of depression was assessed using the German version of the Beck Depression Inventory (Beck et al., 1996). The BDI is a self-report inventory with 21 questions about emotional, cognitive and physical symptoms of depression assessed on a 4-point Likert Scale with good test-retest reliability (Kühner et al., 2007). Anxiety sensitivity was assessed using an abbreviated version of the Anxiety Sensitivity Index (ASI-3; Taylor et al., 2007) with 16-item assessing fear of symptoms associated with sympathetic activation (e.g. elevated heartbeat) on a 5-point Likert Scale.

\subsubsection{Salivary cortisol sampling procedure and assay}

Salivary free cortisol was sampled using Salivette ${ }^{\circledR}$ devices (Sarstedt AG \& Co, Sarstedt, Germany). To determine the exact time of sample collection, Salivettes given to the participants were kept inside vials of the medication event monitoring system (MEMS ${ }^{\circledR}$, AARDEX Group, Sion, Switzerland) and date and time of their opening and closing was automatically recorded by the accompanying TrackCap ${ }^{\circledR}$ via integrated microcircuits. Data was read out from the TrackCap at the time of sample return. In addition, participants noted sampling time and any unusual / special occurrences, together with menstrual cycle information in the case of female subjects, on the protocol sheet filled out on each testing day. After sampling, participants were instructed to store the collected saliva samples in a dry place away from direct heat sources, such as sunlight, until the return of the samples to the researchers.

Previous studies reported a significant increase of subjects' compliance by using electronic monitoring devices and by explaining subjects how these devices verify their self-reported sampling times, even if dummy devices are used (Kudielka et al., 2003). The importance of collecting saliva samples immediately after awakening was highly emphasized by the experimenter.

Biochemical analysis of the saliva samples for cortisol levels was performed at the laboratory of the University of Trier, Germany. Cortisol samples were stored at $-20^{\circ}$ until final analysis. Prior to analysis, samples were thawed and centrifuged at $3000 \mathrm{rpm}$ for $6 \mathrm{~min}$. Saliva cortisol determination was completed using a reliable and validated fluorescence immunoassay with proven validity and reliability (Dressendörfer et al., 1992).

\subsection{Data preprocessing and statistical analysis}

Data preprocessing of the cortisol data included winsorizing of outliers and interpolation of missing values after visual inspection of raw data. Recorded times from the MEMS caps were checked against the times written down on the protocol sheets to allow identification of discrepancies. Special occurrences noted on the protocol sheets like heavy exercise or sickness were used to discard individual observations. For calculation of the conventional CAR, the area under the curve with 
respect to increase (AUCi, Pruessner et al., 2003) was computed for the first $60 \mathrm{~min}$. As comparison with markers of change was the focus of the current paper, we limited our approach to the AUCi - in chronobiological terms, the level at which change takes place is typically discarded, hence a comparison of AUCi with these parameters seemed unnecessary. In the context of the current paper, we refer to the standard CAR AUCi measure as AUCi(CAR). To optimize the description of cortisol pulsatility, four additional parameters were calculated for every distinct pulse seen over the first $270 \mathrm{~min}$ after awakening, for every subject and every day individually, after detrending the data for circadian decline (see Fig. 1). The first measure, called AUCi(CAP), was computed as AUCi taking into account the total number of cortisol samples depicting the first pulse after awakening within each individual. As the individual duration of the CAP varied in length, this measure included all cortisol samples for each individual, which ranged from from waking to the first trough. For every following pulse, the AUCi included all cortisol samples ranging from one trough to the next. The second measure, the amplitude, was defined as the distance from peak value of the current pulse to the (detrended) mesor. The third measure, called peak-to-valley value, was defined as the distance from the peak value of the individual pulse to the successive trough (detrended). Last, we computed the duration of each pulse in minutes, defined as the time (in minutes) from one trough to the next, or in the case of the first pulse, from waking to the first trough.

If a participant's cortisol level showed a descending trend in the first two measures a pulse could still be computed by mirroring along the vertical axis. However, for comparisons of the CAR with the CAP, i.e. AUCi(CAR) versus AUCi(CAP), those cases with a descending trend after awakening were removed from the comparison to not introduce bias in the analysis.

To allow focusing on the physical characteristics of cortisol pulsatility and increase statistical power, especially for comparison of amplitude, duration and AUCi(CAP), with AUCi(CAR), we investigated the two days separately, resulting in a total sample of 101 observations after excluding one observation at the second day of sampling due to insufficient quantity of saliva. To allow examination of cortisol pulsatility during the first $270 \mathrm{~min}$ after awakening in relation to menstrual cycle phase and demographic characteristics, we performed correlational analyses, t- and F-tests by comparing men, women with oral contraceptives, and women in their luteal versus women in their follicular phase. Type III ANOVAs were computed if necessary to control for unbalanced groups.

As a study protocol with sampling intervals of $30 \mathrm{~min}$ does not allow for precise inferences about cortisol levels between two intervals, we estimated the probability of missing a peak between two intervals mathematically. To this end, maximum cortisol increase and maximum cortisol decrease were estimated for each time of measure before, during, and after a measured peak employing an estimated maximum cortisol increase after awakening of ca. $30 \mathrm{nmol} / 1$ as reported by Wuest et al. (2000), together with the cortisol half-life estimates of $28 \mathrm{~min}$ reported by Trifonova et al. (2013). Intersections of increase and accordingly decrease functions and the respective highest value measured were used to estimate a confidence interval for each observation in which a potentially missed peak could have happened. Mathematically estimated confidence intervals for observations with plausible endpoints were summarized by mean values grouped by observed time of cortisol peaks. All statistical analyses were completed using R (version 3.4.2; R Development Core Team, Vienna, Austria), including the packages (car, psych, reshape2, pracma, Hmisc, ggplot2).

\section{Results}

Analysis of data on the overall adherence to the study protocol as monitored with MEMS ${ }^{\circledast}$ vials and compared to the protocol sheets indicated good compliance. One-sample t-tests revealed small but significant deviations from the cortisol sample timing schedule. The mean time of the total sampling period was significantly greater than $270 \mathrm{~min}$ (mean total duration $=275.2 \mathrm{~min}, 95 \%$ CI $[271.5,278.9] ; t$ $(100)=2.79, p=.006)$ and the mean absolute deviation from the $30 \mathrm{~min}$ interval between samples significantly differed from 0 (mean absolute deviation $=3.5 \mathrm{~min}, 95 \%$ CI $[3.1,3.9] ; t(1008)=15.88$, $p<.001$ ). According to the consensus guidelines for the CAR (Stalder et al., 2016), deviations of up to five minutes are acceptable. We reran all analyses after exlcuding subjects with a deviation of the sampling times greater than five minutes and achieved the same results; thus, we kept those samples in the analysis, and thus no further adjustment of the data was undertaken. The estimation of menstrual cycle resulted in four groups: $n=39$ observations for women using OC (mean age $21.4 \pm 2.8$ years; $\mathrm{BDI}=6.0 \pm 4.3$ ), $n=19$ observations for women in the follicular phase (mean age $21.1 \pm 3.4$ years; $\mathrm{BDI}=4.2 \pm 3.8$ ), and $n=19$ observations for women in the luteal phase of their cycle (mean age $21.1 \pm 4.2$ years; $\mathrm{BDI}=8.8 \pm 5.0$ ), and $n=20$ observations for male subjects (mean age $24.9 \pm 2.9$ years; $\mathrm{BDI}=4.6 \pm 3.9$ ). Two subjects were not able to provide information on their menstrual cycle, thus those were excluded from analyses investigating effects of hormonal status. The four resulting groups differed significantly in terms of age $(\mathrm{F}(393)=6.69, p<.001$; with male subjects being significantly older compared to all other groups) and $\mathrm{BDI}(\mathrm{F}(393)=4.59$, $p=.005$; with women in the luteal phase reporting more depressive symptoms compared to men and women in the follicular phase, but no differences for any other post hoc comparison), but not for BMI and ASI. All analyses were calculated with age and BDI as co-variables. As no differences between models with and without co-variables were found, results are reported for the more frugal models without covariates only.

A significant elevation of salivary cortisol within the first $60 \mathrm{~min}$ after awakening was observed, revealing the typical and traditional CAR pattern (mean CAR as defined by AUCi(CAR) $=183.6$, mean increase 106\%). 19 observations had a peak at the time of the first measure and descended thereafter; these observations were included for calculations of the AUCi(CAP) but excluded from the correlational analysis with the AUCi(CAR).

Determination of the absolute length of the first pulse revealed a mean duration of $108 \mathrm{~min}$, significantly longer than $60 \mathrm{~min}$ (one sample t-test: $t(100)=9.67, p<.001,95 \%$ CI [98.2, 118.0], range 60-240 minutes). From the total sample, $36 \%$ of observations showed a duration of $60 \mathrm{~min}$ for the first cortisol pulse after awakening. In $23 \%$ the duration was $90 \mathrm{~min}$, while for another $30 \%$ of observations the first pulse lasted $120 \mathrm{~min}$ (for more details see Table 1).

The cortisol peak occurred at $30 \mathrm{~min}$ after awakening in $43 \%$ of observations. In $33 \%$ of observations the peak was observed at $60 \mathrm{~min}$, while in $6 \%$ of observations it occurred at $90 \mathrm{~min}$. Complete details on mean duration and time of peak of the first pulse are also shown in Table 1. The absolute cortisol levels during the first $270 \mathrm{~min}$ after

Table 1

Distribution of first pulse duration and timing of peak in percent of total observations $(N=101)$. Percentage points do not accumulate to 100 due to rounding up.

\begin{tabular}{|c|c|c|c|c|c|c|c|}
\hline Pulse duration in minutes & 60 & 90 & 120 & 150 & 180 & 210 & 240 \\
\hline Total $(N=101)$ & 36 & 23 & 13 & 11 & 12 & 4 & 2 \\
\hline OC $(n=39)$ & 18 & 9 & 5 & 3 & 2 & 1 & 1 \\
\hline Follicular $(n=19)$ & 8 & 4 & 2 & 2 & 1 & 2 & 0 \\
\hline Luteal $(n=19)$ & 5 & 3 & 3 & 3 & 5 & 0 & 0 \\
\hline Male $(n=20)$ & 3 & 6 & 3 & 3 & 4 & 0 & 1 \\
\hline $\begin{array}{l}\text { Timing of first peak } \\
\text { (minutes since awakening) }\end{array}$ & & 0 & & 30 & \multicolumn{2}{|c|}{60} & 90 \\
\hline Total $(N=101)$ & & 19 & & 43 & \multicolumn{2}{|c|}{33} & 6 \\
\hline OC $(n=39)$ & & 5 & & 21 & \multicolumn{2}{|c|}{9} & 4 \\
\hline Follicular $(n=19)$ & & 5 & & 4 & \multicolumn{2}{|c|}{8} & 2 \\
\hline Luteal $(n=19)$ & & 2 & & 6 & \multicolumn{2}{|c|}{11} & 0 \\
\hline Male $(n=20)$ & & 6 & & 10 & \multicolumn{2}{|c|}{4} & 0 \\
\hline
\end{tabular}




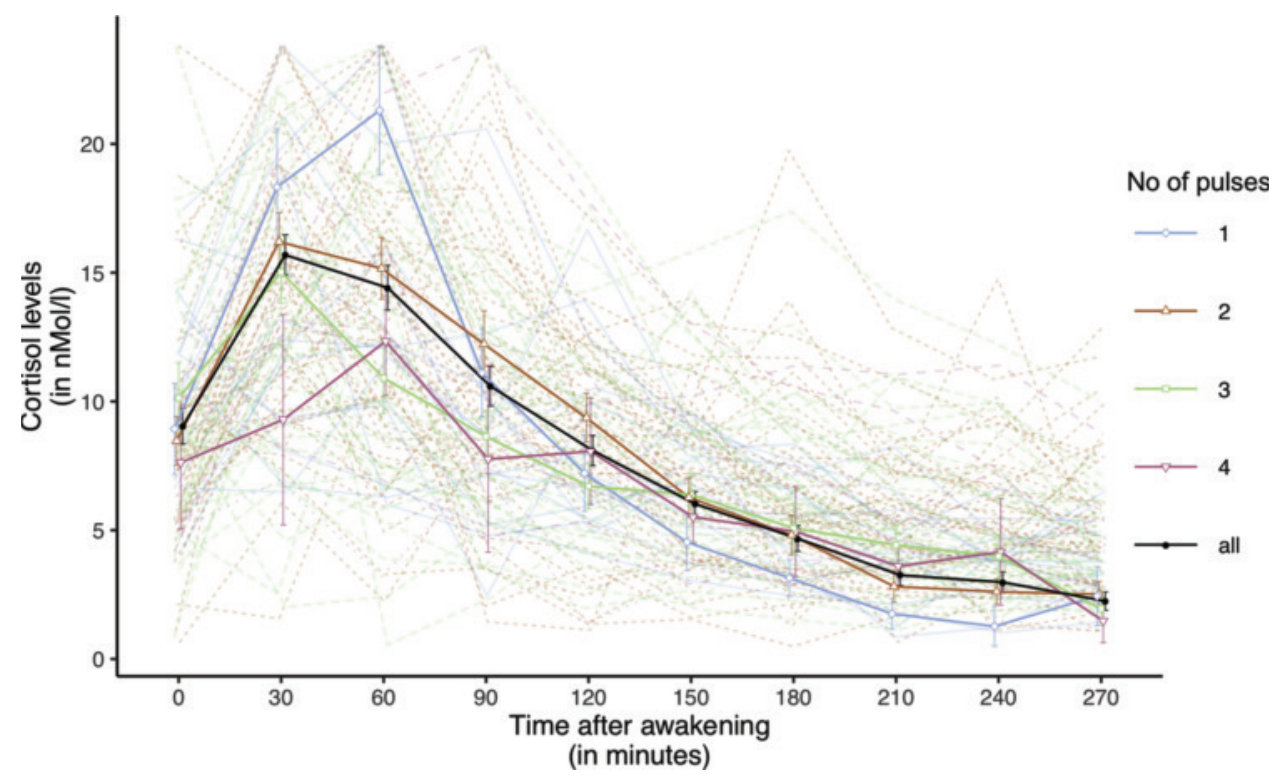

Fig. 2. Absolut cortisol levels for all observations and mean cortisol levels grouped by observed number of pulses during the first 270 min after awakening before detrending.

awakening can be drawn from Fig. 2 depicting mean values over time for all observations and separated by groups according to the number of pulses observed during the sampling period.

Correlations between the AUCi(CAR) and the AUCi(CAP) were high, shown in Table 2 and illustrated in Fig. 3. R-square (the amount of explained variance) varied between $49 \%$ and $10 \%$, suggesting that between $50 \%$ and $90 \%$ of variance among the various measures remained unexplained. More importantly, a systematic contortion appeared when observing the results separately for the hormonal status groups intermixed with gender in our sample. In men, the slope of the correlation of the AUCi(CAR) with the AUCi(CAP) is shallower, while in women, the slope of the correlation is steeper, suggesting that stronger increases in the AUCi(CAR) were associated with stronger increases in the AUCi(CAP). This interaction of hormonal status and gender with the slope was significant $(F(393)=5.86, p=.017$; Fig. 3).

When looking at pulse duration, additional differences between these groups emerged. In our sample, men showed a significantly longer duration of the first pulse than women with $124.5 \mathrm{~min}$ for men versus $96.2 \mathrm{~min}$ for women taking OC, 104.2 for women in the follicular phase, and $120 \mathrm{~min}$ for women in the luteal phase $(F(195)=5.80 p=.018)$. Investigating the peak-to-valley measure, differences between groups emerged as well: for the first pulse, women taking OC showed the smallest peak-to-valley value while women in the luteal phase showed the largest measure (5.3 vs $7.8, F(195)=4.39, p=.039$ ). For the second pulse of the day, the opposite was the case, with women taking OC showing the highest peak-to-valley measure and women in the luteal phase the smallest (4.8 vs. 2.7, $F(182)=5.18 p=.025)$. Differences in duration and relative increase and decline of cortisol release are depicted in Fig. 4.

Thus, contrary to our hypothesis, a longer first pulse appears to be

Table 2

Associations between first and subsequent pulses in different parameters describing cortisol release.

\begin{tabular}{|c|c|c|c|c|c|c|c|c|}
\hline \multirow[b]{2}{*}{ Variable } & \multicolumn{4}{|c|}{ First to second pulse } & \multicolumn{4}{|c|}{ Second to third pulse } \\
\hline & $r$ & $t$ & $d f$ & $p$ & $r$ & $t$ & $d f$ & $p$ \\
\hline AUCi & -.20 & -1.90 & 85 & .061 & .35 & 2.30 & 38 & .027 \\
\hline Amplitude & -.21 & -1.99 & 85 & .050 & .25 & 1.61 & 38 & .115 \\
\hline Peak-to-valley & -.30 & -2.91 & 85 & .005 & .21 & 1.33 & 38 & .192 \\
\hline
\end{tabular}

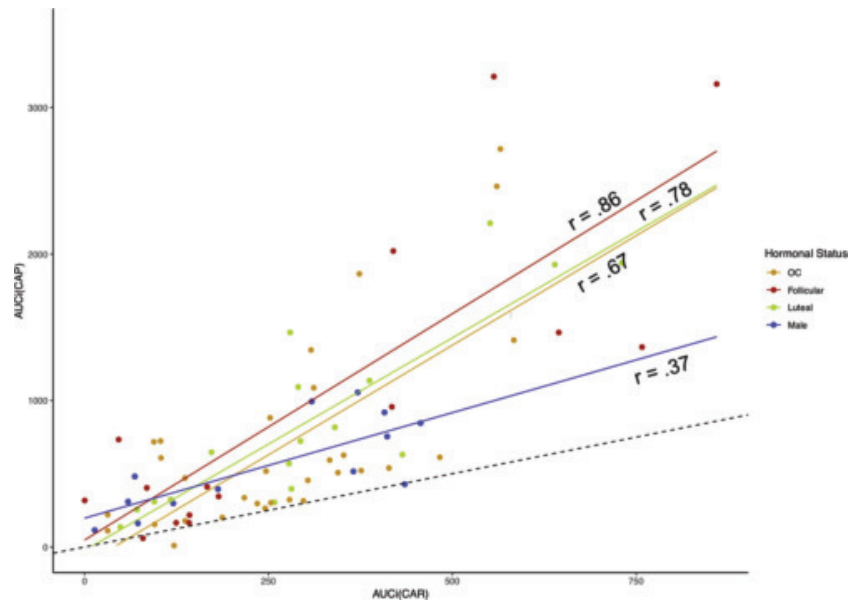

Fig. 3. Scatterplot of the correlation between CAR calculated as AUCi(CAR), over $60 \mathrm{~min}$ and AUCi(CAP), based on the assumed length of pulse taking into account hormonal status (luteal phase, follicular phase, use of oral contraceptives), and gender. Subjects with negative AUCi(CAR) were excluded from this analysis.

associated with a weaker second pulse when taking into account gender and estimated menstrual cycle phase $(F(281)=4.09, p=.047)$. These differences could not be observed when assessing the first pulse using the traditional CAR formula (AUCi(CAR) $(F(195)=.58, p=.45$ ).

These relationships are also visible when investigating intercorrelations among these factors (see table 3). The first pulse was negatively associated with the second pulse, whereas a positive association between the second and third pulse was observed.

Finally, while $40 \%$ of daily observations consisted of three to five pulses, around $47 \%$ consisted of two pulses, and $14 \%$ showed only one pulse over the $270 \mathrm{~min}$ assessment period. No other associations among the variables sex, age, BDI, ASI, or BMI and parameters describing cortisol release during the first $270 \mathrm{~min}$ after awakening were found.

Further exploratory analysis revealed that, according to the modulated estimations of potentially missed peaks, mean confidence interval for peak time in $n=41$ (out of 43) observations with a measured peak at $30 \mathrm{~min}$ after awakening is at 27.59-35.27 (or 7.83-46.83) minutes, in $n=31$ (out of 33) observations with a measured peak at $60 \mathrm{~min}$ after 

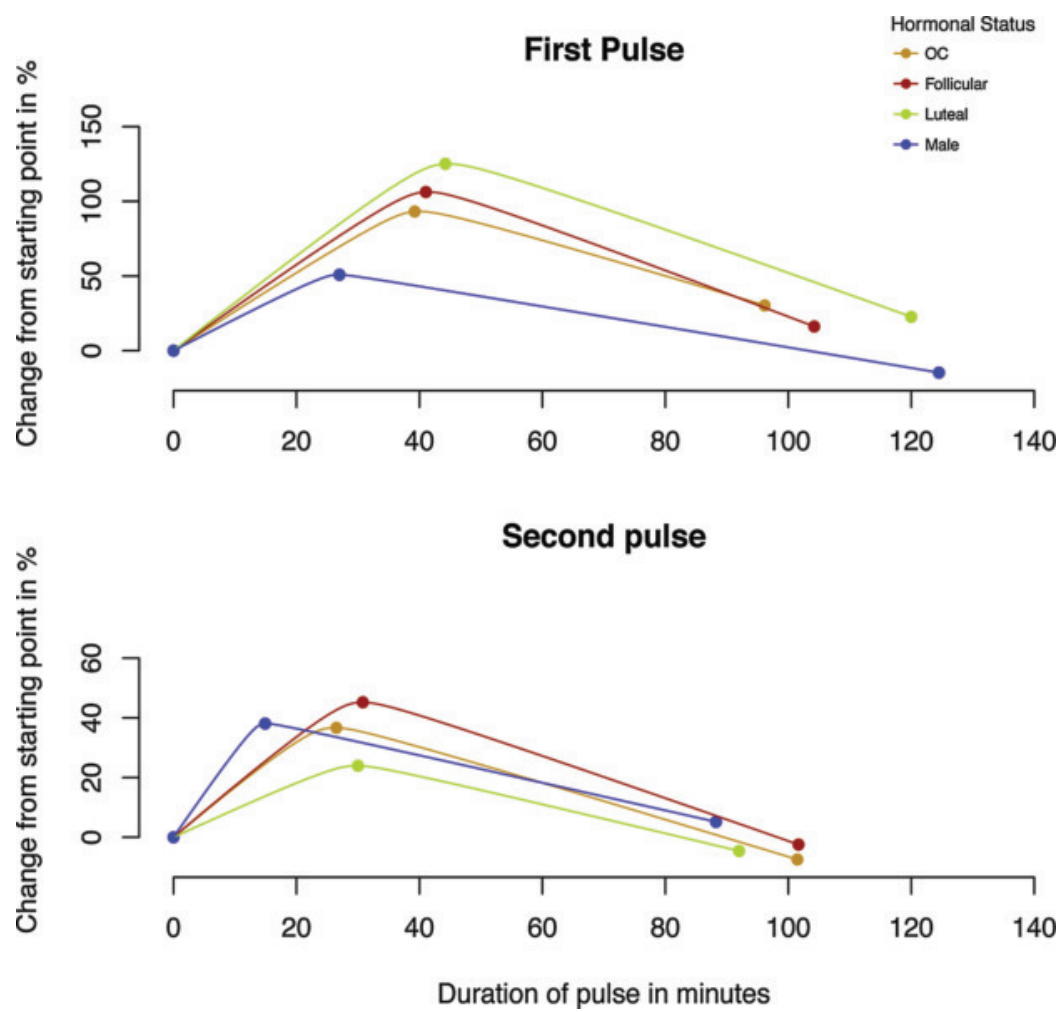

Fig. 4. Percentage of increase and decrease of cortisol release during the first and second pulse relative to the respective starting point with respect to hormonal status.

awakening at 44.01-62.31 (or 34.42-72.75) minutes, and in $n=5$ (out of 6) observations with a measured peak at 90 min after awakening at 71.39-91.48 (or 64.81-101.04) minutes.

\section{Discussion}

With the aim of investigating the dynamic regulation of cortisol pulsatility beyond awakening, the present study measured salivary cortisol for the first $270 \mathrm{~min}$ after awakening in intervals of $30 \mathrm{~min}$ in a sample of healthy young men and women with a total sample of 101 observations. A significant increase of salivary cortisol was observed after awakening followed by up to four additional pulses over the 270 min observation period. We could demonstrate that the mean duration of the cortisol awakening pulse (CAP) was $108 \pm 50 \mathrm{~min}$ for the entire sample. First, we would like to point out the high variability in the duration of the CAP with a range from 60 to $240 \mathrm{~min}$. Secondly, the CAP was completed after $60 \mathrm{~min}$ in about a third (36\%) of observations only, with the majority of CAPs lasting significantly longer. Thus, when measured for $60 \mathrm{~min}$, the recovery phase of the first rise of cortisol in the morning is missed in most cases, and even the peak could be missed in some cases. The review paper by Koolhaas et al. (2011) specifically discussing cortisol response dynamics suggests to put more emphasis on the recovery phase to avoid missing important group differences. What - if any - health, stress or lifestyle characteristics might be associated with the recovery phase is at this point pure speculation, since our study did not include such information, but appears as an interesting object of investigation for future studies. There are studies suggesting that the cortisol decline during recovery does have a distinct share in explanation of variance of psychological variables (Miller et al., 2007).

Meanwhile, the observed time points of peaks are in line with the literature on CAR, reporting decreasing cortisol levels with the beginning of the first measure in about $13-20 \%$ of observations (Stalder et al., 2016) and later peaks at 75 or $90 \mathrm{~min}$ in about $10 \%$ of observations (Trifonova et al., 2013).

Significant associations with pulse dynamics emerged from taking into account gender and estimated menstrual cycle phase, i.e. separating the sample into men and women in the luteal or follicular phase, or women taking OC. Women who assumably had higher levels of circulating estrogen, i.e. women in the follicular phase and OC users, showed a shorter first pulse and a stronger second pulse. In line with previous research on estradiol- associated changes in cortisol regulation (Kudielka et al., 2009; Kumsta et al., 2007), our results suggest differential cortisol release with respect to hormonal status for the CAP. Previous studies had also shown significant associations between hypothalamic-pituitary-gonadal axis activity and the timing and characteristics of the CAR: women after menarche show a peak $45 \mathrm{~min}$ after awakening (Oskis et al., 2009), and women across the menstrual cycle phase show distinct CAR profiles (Wolfram et al., 2011).

At least in the current sample, the differences we observed using the revised pulse computations did not emerge when looking for group differences with the traditional CAR measure, suggesting increased sensitivity to capture hormonal interactions with the CAP when investigating the awakening pulse for its entire duration as compared to the 60-minute time period.

With regard to ultradian pulsatility, our data revealed a negative association between the first and second pulse and a positive association between the second and third pulse. We had hypothesized that the CAP would be significantly positively associated with subsequent pulses of cortisol, following the argumentation of Strüber et al. (2014). The current data does not support this assumption; however, it is important to point out the possibility that the CAR as well as the CAP are simply different measures of ultradian HPA axis regulation. Buchanan et al. (2004) could previously demonstrate that hippocampal lesions are associated with an absent CAR, which suggests that the awakening response is distinct from ultradian regulation (Buchanan et al., 2004). This is also in line with Stalder et al. (2016), who suggested a distinct nature of the CAR within ultradian regulation of cortisol. 
While only for 40 observations a complete third pulse could be recorded, the finding of a significantly positive correlation between the second and the third pulse supports the assumption of a positive correlation among pulses, as stated in the model by Strüber et al., who emphasized that the negative feedback of cortisol of the current pulse could contribute to the stimulation of the next pulse via hippocampal interneurons. Thus, the findings from the current study support the idea that the CAP is a distinct feature of HPA axis activity, and not part of ultradian regulation.

Albeit preliminary, the current results allow for a few recommendations for future CAR and CAP research. To begin with, the finding of the first pulse being substantially longer than 60 min suggests that the current practice of assessing the CAR for a duration of no more than 60 min might not be ideal for all studies and research questions as it does not allow for additional information provided by the fall from peak to trough. While admittedly adding cost and inconvenience, a sample at 90 and 120 min would allow to capture the complete duration of the CAP in the vast majority of subjects and thus a more comprehensive estimation of the amount of cortisol present in saliva after awakening. What is important to note here is the observation that simply calculating the AUCi over 90 or 120 min alone would not add useful information: since individual pulses vary in length, it is instead necessary to establish the exact duration of the CAP individually for each subject, and then calculate the AUCi(CAP) accordingly. This would result in a dataset where the number of samples used for the calculation of the CAP would vary for each subject. In a review on cortisol pulsatility, Young et al. (2004) discussed the intrinsic rhythmicity of HPA axis activity. The authors also recommended to measure cortisol for longer periods of time to avoid loss of information and 'blurring' of data when averaging across peaks and troughs. Although this was based mainly on rodent studies and without precise assumptions about timing and duration of pulses, it did emphasize the idea of ultradian pulsatility. Trifonova et al. (2013) built on this recommendation and analyzed cortisol data for assessment of ultradian cortisol profiles in men for $8 \mathrm{~h}$, in 15-minute intervals in both blood and saliva, demonstrating the plausibility of this approach.

An important finding from the current study is that the conventional CAR calculation over $60 \mathrm{~min}$ is very different from the complete first pulse of cortisol after awakening (i.e. the CAP), for the majority of subjects. As the current sample consisted of young healthy subjects only, it is unclear whether duration and amplitude of the CAP is systematically linked to clinical and psychopathological factors. Thus, by extending sampling periods in future studies evidence could be obtained to reveal such associations.

A number of significant limitations need to be acknowledged. First, in the current study, we measured salivary cortisol in 30-minute intervals which might be considered too long a time period between samples to accurately determine individual pulses. As we set out to expand the current practice of CAR research, a sampling protocol which is used in more than $70 \%$ of studies between 2013-2014 with 3 or less samples during the first $1 \mathrm{~h}$ after awakening (Stalder et al., 2016) appeared feasible. However, precise information about the peak might be lost, or individual pulses even be overlooked. Previous studies have shown that half-life of salivary cortisol is about $30 \mathrm{~min}$ (Trifonova et al., 2013) and that after stimulation it takes between 20-30 minutes for cortisol to reach its peak (Engert et al., 2011; Wuest et al., 2000). However, in accordance with these studies and the reported confidence intervals for peaks as measured in this sample, we still feel that the finding of the first complete pulse after awakening exceeding $60 \mathrm{~min}$ in a meaningful pattern still stands out. Nonetheless, the 30-minute interval is not ideal and will likely have led to missing the exact peak of the awakening pulse in many cases, especially in women, who are reported to have their mean peak at 45 min once menarche is reached (Oskis et al., 2009). The reason for selecting the current design was related to feasibility; when piloting the design in a small sample, a 15minute interval for $270 \mathrm{~min}$ was perceived as rather disruptive and not feasible for subjects to follow.

Further, while the use of MEMS caps has to be considered a strength, lack of objective assessment of wake time has to be considered a limitation. For optimal monitoring of sampling times and adherence to the study protocol, a combination of MEMS caps together with an objective recording of awakening, through for example use of Actigraph would have been ideal. By this means, control of compliance and at the same time high ecological validity due to the domestic setting could have been ensured (cf. Stalder et al., 2016). Further, a detailed protocol of a participant's day (e.g. food and exercise logs), combined with a more frequent sampling method, could have provided a more accurate representation of subjects' HPA axis activity. While we recommend these steps, ecological and financial constraints prevented us from employing them in the current study.

Furthermore, heterogeneity of this small sample in terms of sex, OC use, and menstrual cycle has to be considered as a limitation. Larger samples with accurate measurements of confounding hormone levels, e.g. assessing surge of luteinizing hormone as defined by a chromatographic ovulation predictor test kit (e.g. Wolfram et al., 2011), should be collected to confirm and extend our findings. Future research should take these limitations into consideration, and improve upon the experimental design when financially and ecologically feasible.

Taken together, the cortisol awakening response measured over a 60-minute period provides important information about HPA axis regulation which we suggest can be extended to $120 \mathrm{~min}$ to capture the cortisol awakening pulse, which potentially reveals additonal associations with psychological and clinical factors. In our study of healthy young men and women, assocations with sex and estimated hormonal status of the women emerged when analysing the AUCi of the CAP, and the length of the first pulse. To account for the complete CAP in the majority of cases, we recommend an extension of the assessment period to $120 \mathrm{~min}$.

\section{Conflict of interest}

None of the authors report biomedical financial interests or other potential conflicts of interest.

\section{References}

Beck, A.T., Steer, R.A., Brown, G.K., 1996. BDI-II, Beck Depression Inventory.

Buchanan, T.W., Kern, S., Allen, J.S., Tranel, D., Kirschbaum, C., 2004. Circadian regulation of cortisol after hippocampal damage in humans. Biol. Psychiatry 56, 651-656. https://doi.org/10.1016/j.biopsych.2004.08.014.

Dedovic, K., Ngiam, J., 2015. The cortisol awakening response and major depression: examining the evidence. Neuropsychiatr. Dis. Treat. 11, 1181-1189. https://doi.org/ 10.2147/NDT.S62289.

Dressendörfer, R.A., KIirschbaum, C., Rohde, W., Stahl, F., Strasburger, C.J., 1992. Synthesis of a cortisol biotin conjugate and evaluation as a tracer in an immunoassay for salivary cortisol measurement. J. Steroid Biochem. Mol. Biol. 43, 683-692.

Engert, V., Vogel, S., Efanov, S.I., Duchesne, A., Corbo, V., Ali, N., Pruessner, J.C., 2011. Investigation into the cross-correlation of salivary cortisol and alpha-amylase responses to psychological stress. Psychoneuroendocrinology 36, 1294-1302. https:// doi.org/10.1016/j.psyneuen.2011.02.018.

Gwirtsman, H.E., Kaye, W.H., George, D.T., Jimerson, D.C., Ebert, M.H., Gold, P.W., 1989. Central and peripheral acth and cortisol-levels in Anorexia-Nervosa and bulimia. Arch. Gen. Psychiatr. 46, 61-69.

Kirschbaum, C., Hellhammer, D.H., 1989. Salivary cortisol in psychobiological research an overview. Neuropsychobiology 22, 150-169.

Koolhaas, J.M., Bartolomucci, A., Buwalda, B., de Boer, S.F., Flügge, G., Korte, S.M., Meerlo, P., Murison, R., Olivier, B., Palanza, P., Richter-Levin, G., Sgoifo, A., Steimer, T., Stiedl, O., van Dijk, G., Wöhr, M., Fuchs, E., 2011. Stress revisited: a critical evaluation of the stress concept. Neurosci. Biobehav. Res. 35, 1291-1301. https:// doi.org/10.1016/j.neubiorev.2011.02.003.

Kudielka, B.M., Broderick, J.E., Kirschnaum, C., 2003. Compliance with saliva sampling protocols: electronic monitoring reveals invalid cortisol daytime profiles in noncompliant subjects. Psychosom. Med. 65 (2), 313-319.

Kudielka, B.M., Hellhammer, D.H., Wüst, S., 2009. Why do we respond so differently? Reviewing determinants of human salivary cortisol responses to challenge. Psychoneuroendocrinology 34, 2-18. https://doi.org/10.1016/j.psyneuen.2008.10. 004.

Kühner, C., Bürger, C., Keller, F., Hautzinger, M., 2007. [Reliability and validity of the revised Beck Depression Inventory (BDI-II). Results from German samples]. 
Nervenarzt 78, 651-656. https://doi.org/10.1007/s00115-006-2098-7.

Kumari, M., Chandola, T, Brunner, E., Kivimaki, M., 2010. A nonlinear relationship of generalized and central obesity with diurnal cortisol secretion in the whitehall II study. J. Clin. Endocrinol. Metab. 95, 4415-4423. https://doi.org/10.1210/jc.20092105.

Kumsta, R., Entringer, S., Hellhammer, D.H., Wüst, S., 2007. Cortisol and ACTH responses to psychosocial stress are modulated by corticosteroid binding globulin levels Psychoneuroendocrinology 32, 1153-1157. https://doi.org/10.1016/j.psyneuen. 2007.08.007.

Lenton, E.A., Landgren, B.M., Sexton, L., 1984. Normal variation in the length of the luteal phase of the menstrual cycle: identification of the short luteal phase. Br. J. Obstretrics Gynecology 91, 685-689.

Lightman, S.L., Conway-Campbell, B.L., 2010. The crucial role of pulsatile activity of the HPA axis for continuous dynamic equilibration. Nat. Publishing Group 11, 710-718. https://doi.org/10.1038/nrn2914.

Mastorakos, G., Ilias, I., 2003. Maternal and fetal hypothalamic-pituitary-adrenal axes during pregnancy and postpartum. Women's Health Dis.: Gynecol. Reprod. Issues 997, 136-149. https://doi.org/10.1196/annals.1290.016.

Merswolken, M., Deter, H.-C., Siebenhuener, S., Orth-Gomér, K., Weber, C.S., 2012. Anxiety as predictor of the cortisol awakening response in patients with coronary heart disease. Int. J. Behav. Med. 20, 461-467. https://doi.org/10.1007/s12529-0129233-6.

Miller, G.E., Chen, E., Zhou, E.S., 2007. If it goes up, must it come down? Chronic stress and the hypothalamic-pituitary-adrenocortical axis in humans. Fam. Psychol. 133 25-45. https://doi.org/10.1037/0033-2909.133.1.25.

Oskis, A., Loveday, C., Hucklebridge, F., Thorn, L., Clow, A., 2009. Diurnal patterns of salivary cortisol across the adolescent period in healthy females. Psychoneuroendocrinology 34, 307-316. https://doi.org/10.1016/j.psyneuen.2008. 09.009.

Pruessner, J.C., Wolf, O.T., Hellhammer, D.H., Buske-Kirschbaum, A., Auer von, K., Jobst, S., Kaspers, F., Kirschbaum, C., 1997. Free cortisol levels after awakening: a reliable biological marker for the assessment of adrenocortical activity. Life Sci. 61, 2539-2549.

Pruessner, J.C., Kirschbaum, C., Meinlschmid, G., Hellhammer, D.H., 2003. Two formulas for computation of the area under the curve represent measures of total hormone concentration versus time-dependent change. Psychoneuroendocrinology 28, 916-931. https://doi.org/10.1016/S0306-4530(02)00108-7.
Pruessner, M., Vracotas, N., Joober, R., Pruessner, J.C., Malla, A.K., 2013. Blunted cortisol awakening response in men with first episode psychosis: relationship to parental bonding. Psychoneuroendocrinology 38, 229-240. https://doi.org/10.1016/j. psyneuen.2012.06.002

Rivest, R.W., Schulz, P., Lustenberger, S., Sizonenko, P.C., 1989. Differences between circadian and ultradian organization of cortisol and melatonin rhythms during activity and rest. J. Clin. Endocrinol. Metab. 68, 721-729. https://doi.org/10.1210/ jcem-68-4-721.

Scheurink, A.J., Ammar, A.A., Benthem, B., Van, D.G., Sodersten, P.A., 1999. Exercise and the regulation of energy intake. Int. J. Obes. Relat. Metab. Disord. 23 (S3), S1-S6.

Stalder, T., Kirschbaum, C., Kudielka, B.M., Adam, E.K., Pruessner, J.C., Wüst, S., Dockray, S., Smyth, N., Evans, P., Hellhammer, D.H., Miller, R., Wetherell, M.A., Lupien, S.J., Clow, A., 2016. Assessment of the cortisol awakening response: expert consensus guidelines. Psychoneuroendocrinology 63, 414-432. https://doi.org/10. 1016/j.psyneuen.2015.10.010.

Strüber, N., Strüber, D., Roth, G., 2014. Impact of early adversity on glucocorticoid regulation and later mental disorders. Neurosci. Biobehav. Res. 38, 17-37. https:// doi.org/10.1016/j.neubiorev.2013.10.015.

Taylor, S., Zvolensky, M.J., Cox, B.J., Psychological, B.D., 2007. Robust dimensions of anxiety sensitivity: development and initial validation of the Anxiety Sensitivity Index-3. n.d.. Psychol. Assess. 19, 176.

Thomas, L., 1992. Labor \& diagnose: indikation und Bewertung Von Laborbefunden in der medizinischen diagnostik. Medizinische Verlags Gesellschaft, Marburg.

Trifonova, S.T., Gantenbein, M., Turner, J.D., Muller, C.P., 2013. The use of saliva for assessment of cortisol pulsatile secretion by deconvolution analysis.

Psychoneuroendocrinology 38, 1090-1101. https://doi.org/10.1016/j.psyneuen. 2012.10.016.

Wolfram, M., Bellingrath, S., Kudielka, B.M., 2011. The cortisol awakening response (CAR) across the female menstrual cycle. Psychoneuroendocrinology 36 (6), 905-912. https://doi.org/10.1016/j.psyneuen.2010.12.006.

Wuest, S., Wolf, J., Hellhammer, D.H., Federenko, I., Schommer, N., Kirschbaum, C. 2000. The cortisol awakening response - normal values and confounds. Noise Health 2, 79-88.

Young, E., Abelson, J., Lightman, S., 2004. Cortisol pulsatility and its role in stress regulation and health. Front. Neuroendocrin 25, 69-76. https://doi.org/10.1016/j. yfrne.2004.07.001. 